Article

\title{
Influence of Control Structures and Load Parameters on Performance of a Pseudo Direct Drive
}

\author{
Mohammed Bouheraoua ${ }^{1, *}$, Jiabin Wang ${ }^{2}$ and Kais Atallah ${ }^{2}$ \\ 1 Magnomatics Ltd, Park House, Bernard Road, Sheffield S2 5BQ, UK \\ 2 Department of Electronic and Electrical Engineering, The University of Sheffield, Mappin Street, \\ Sheffield S1 3JD, UK; E-Mails: j.b.wang@sheffield.ac.uk (J.W.); k.atallah@sheffield.ac.uk (K.A.) \\ * Author to whom correspondence should be addressed; E-Mail: m.bouheraoua@magnomatics.com; \\ Tel.: +44-114-241-2587; Fax: +44-114-241-2571.
}

Received: 10 February 2014; in revised form: 23 June 2014 / Accepted: 30 June 2014 /

Published: 4 July 2014

\begin{abstract}
The paper describes an in-depth and systematic analysis of a pseudo direct drive permanent magnet machine in closed loop control. Due to the torque being transmitted from the high-speed rotor (HSR) to the low-speed rotor (LSR), through a relatively low stiffness magnetic gear with non-linear characteristics, speed oscillations appear in the drive output with a conventional proportional integral (PI) controller. Therefore two candidate controllers have been proposed as an alternative to the PI control and all controllers have been optimally tuned with a genetic algorithm against a defined criterion. Furthermore, closed loop models are established in the complex frequency domain to determine the system damping and the cause of the oscillations. Consequently, the best controller structure that improves the dynamic behaviour of the system in terms of speed tracking and disturbance rejection could be identified, based on the frequency domain analysis. Experimental results are presented to validate the analysis and the proposed control technique.
\end{abstract}

Keywords: permanent magnet machines; electric drives; genetic algorithm; stability; state feedback controller; pseudo direct drive; PDD

\section{Introduction}

High torque, low speed machines have increasingly been used in wind turbines, ship propulsion systems and traction applications. Various designs and concepts have been employed to achieve high 
torque density or air-gap shear stress [1]. It has been shown that a Pseudo Direct Drive (PDD), realised by mechanical and magnetic integration of a permanent magnet (PM) machine and a magnetic gear, can achieve torque densities in excess of $60 \mathrm{kNm} / \mathrm{m}^{3}$ [2]. In addition, for large PDDs a torque density of $110 \mathrm{kNm} / \mathrm{m}^{3}$ is attainable as reported in [1] with a power factor greater than 0.9 , with low cogging torque and natural air-cooling. In contrast Vernier and Transverse flux PM machines (TFM) [3-5] may exhibit higher torque densities at the expense of an inherently low power factor. Indeed, the power factor of a TFM may be lower than 0.5 , resulting in very large converter volt-ampere (VA) ratings and requiring significant converter overhead.

The PDD alleviates the problems associated with mechanical gearboxes, such as acoustic noise, vibrations, the need for lubrication and maintenance, as well as low reliability due to wear and tear [2].

Figures 1 and 2 show the schematic of a PDD design. The electromagnetic torque is produced by the interaction of the 2 pole-pair permanent magnets $\left(p_{h}=2\right)$ on the high-speed rotor (HSR) with the currents in the stator winding. This torque is transmitted to the low-speed rotor (LSR), with a gear ratio $G_{r}=n_{s} / p_{h}$, by the interaction of the 21 pole-pair $\left(p_{l}=21\right)$ stationary permanent magnets and the 21st space harmonic, which results from the modulation of the 2 pole-pair magnetic field on the HSR by the $23\left(n_{s}=23\right)$ ferromagnetic pole-pieces of the LSR.

Figure 1. Radial cross-section of the Pseudo Direct Drive (PDD).

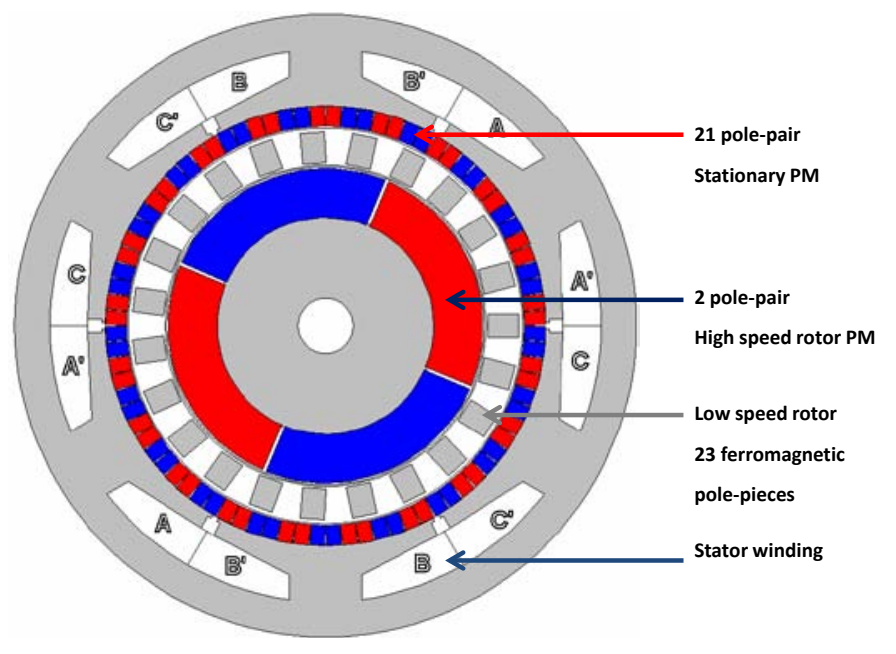

Since the torque is transmitted to the load through a relatively low stiffness magnetic gear, speed and position oscillations are present with conventional PI speed control and better control strategies are required. In addition, although the load is connected to the LSR, accurate position information about the HSR is also required for electronic commutation purposes. Inferring one rotor speed/position to another through the gear ratio alone is not possible due to the factor that the angular displacement between the two rotors varies with transmitted torque.

Furthermore, overload protection is a significant advantage of a PDD, since when subjected to a load torque greater than its pull-out torque, it should harmlessly slip. However, this may lead to a pulsating torque which can induce noise and vibration. Although this may not result in any physical damage, the time taken for the machine to recover and resume normal operation after the transient overload torque disappears, will depend on how fast the slip can be detected and how the control strategy adapt when the transient overload torque occurs [6]. 
Figure 2. Axial cross-section of the PDD.

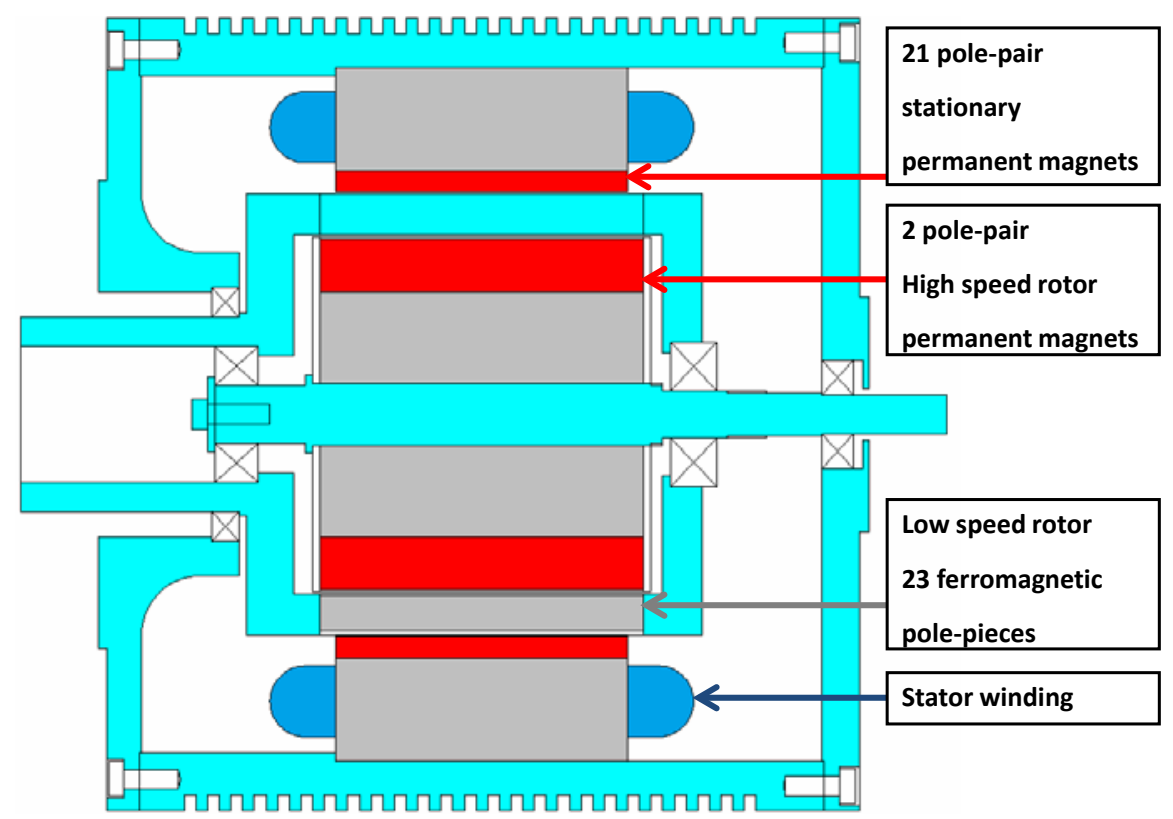

The servo control which includes two inertias connected by a compliant mechanical coupling has been studied in [7-9]. It is shown that the ratio of load inertia to motor inertia directly affects the performance of the controller in terms of suppressing resonance oscillations in the load. A drive train composed of a motor and load connected through a 1:1 magnetic coupling, where the inertia ratio is close to unity, has been reported in [10,11]. Since the magnetic gear is far more (mechanically) flexible than a classical mechanical coupling and has medium torsional stiffness, an integral proportional (IP) controller is employed to reduce the speed oscillations on the load. In contrast the PDD drive train contains not only a low stiffness coupling but also a gearing mechanism which has a significant influence on the damping and the inertia ratio between the two rotors.

Previous work on the PDD control has been reported by Wang and Atallah in [12] where two types of controllers, a PI and a state feedback (SFBK) controller, have been studied. It has been shown that, due to the magnetic gearing and lack of damping, torsional oscillation will result with the PI controller, causing abrupt speed and position transients, increased copper loss in the motor, and poor overall performance. The SFBK tuned according to [13] has improved the performance, but not to the point where the oscillations are completely removed. An observer based SFBK controller has been applied to the PDD control in [14,15]. The feedback gains have been tuned using Genetic Algorithm (GA) [16] to satisfy the integral time multiplied by absolute error (ITAE) criterion given by Equation (1). This performance index has the advantages of producing smaller overshoots and less oscillation than the integral of square error (ISE) or integral of the absolute error (IAE). It is shown that the proposed control technique has significantly reduced torsional oscillations in the output.

$$
I T A E=\int_{0}^{T} t|e(t)| d t
$$

This paper performs complex frequency domain analyses of the PDD in closed loop. The state-space equations of the closed loop system with three candidate controllers, viz., PI, IP and SFBK are established and the resulting eigenvalues are computed and presented in the s-plane. A relationship 
between the poles/zeros and the system states is identified, and the effect of the controller structure on the system damping is quantified. This provides an in-depth understanding of how the controller structure and control/load parameters influence the system damping. An experimental rig has been developed and the simulated results were validated through systematic experimental tests.

\section{Modelling of Pseudo Direct Drive}

In a PDD, the HSR and LSR are magnetically coupled and the mechanical load is applied to the LSR. The torque is transmitted from the HSR to the LSR. The equations that govern their motion are as follows

$$
\begin{gathered}
\frac{d \omega_{h}}{d t}=\frac{T_{e}}{J_{h}}-\frac{T_{\max }}{J_{h} G_{r}} \sin \left(\theta_{e}\right)-\frac{B_{h}}{J_{h}} \omega_{h}-\frac{K_{d}}{J_{h}}\left(p_{h} \omega_{h}-n_{s} \omega_{o}\right) \\
\frac{d \omega_{o}}{d t}=\frac{T_{\max }}{J} \sin \left(\theta_{e}\right)-\frac{B_{o}}{J} \omega_{O}+\frac{K_{d} G_{r}}{J}\left(p_{h} \omega_{h}-n_{s} \omega_{o}\right)-\frac{T_{L}}{J}
\end{gathered}
$$

where $\omega_{h}, J_{h}, B_{h}$ are the angular speed, the moment of inertia and the viscous damping of the HSR respectively, $\omega_{o}, J, B_{o}$ are the angular speed, the combined inertia of the $\operatorname{LSR} J_{o}$ and the load $J_{L}$, and the combined damping coefficient of the LSR and the load respectively. $G_{r}=n_{s} / p_{h}$ is the magnetic gear ratio, where $p_{h}$ is the number of pole-pairs on the HSR and $n_{s}$ is the number of ferromagnetic pole pieces on the LSR. $T_{L}$ is the load torque, $T_{\max }$ is the pull-out torque reflected to the LSR and $T_{e}$ is the electromagnetic torque produced by the q-axis current $i_{q}$, when a surface-mounted magnet topology is employed for the HSR, and is given by

$$
T_{e}=K_{t} i_{q}, \quad K_{t}=\frac{3}{2} p_{h} \varphi_{m}
$$

The stator flux-linkage is $\varphi_{m}$. The electromagnetic torque is transmitted to the LSR via an equivalent magnetic spring with a stiffness given with respect to LSR as

$$
K_{e s}=\frac{\partial T}{\partial \theta_{o}}=n_{s} \times T_{\max } \cos \left(\theta_{e}\right)
$$

The referred angel $\theta_{e}$ is defined as the angular displacement between the HSR and the LSR, given by

$$
\theta_{e}=p_{h} \theta_{h}-n_{s} \theta_{o}
$$

The angular positions of the HSR and LSR are given by $\theta_{h}$ and $\theta_{o}$ respectively. From Equation (5) it can be shown that the system is stable when the stiffness $K_{e s}$ is positive, i.e., only when $\theta_{e}$ is within the range $2 \pi n-\frac{\pi}{2}>\theta_{e}>2 \pi n+\frac{\pi}{2}, n$ is an integer number.

The equivalent inertia of the HSR with the magnetic gear seen by the LSR is obtained by

$$
J_{e}=G_{r}^{2} J_{h}
$$

The damping coefficient $K_{d}$ is associated with the referred angular speed $\frac{d \theta_{e}}{d t}$ between the HSR and LSR due to eddy current loss in the HSR and iron loss in the LSR. Since the damping effect, $K_{d}$ is very small it is assumed that $K_{d}=0$. The nonlinear transfer function block diagram of the PDD is shown in Figure 3. The linearized representation of the PDD transfer function is shown in Figure 3. 
Figure 3. The transfer function block diagram

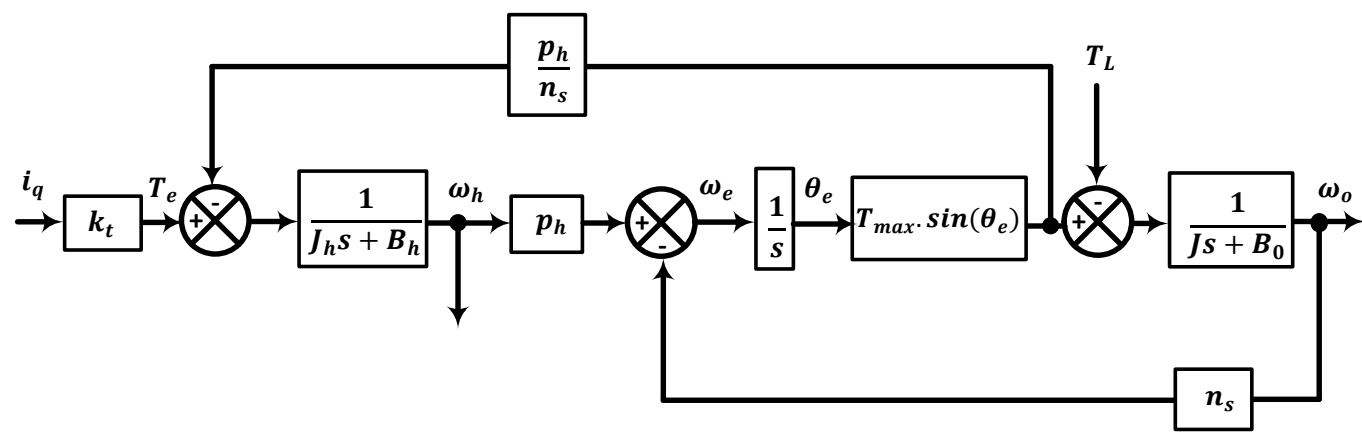

The transfer function of the mechanical system in Figure 3 becomes [12]

$$
\begin{aligned}
& \frac{\omega_{r}(s)}{T_{r}(s)}=\frac{s^{2}+\omega_{a}^{2}}{J_{e} s\left(s^{2}+\omega_{n}^{2}\right)}, \quad \omega_{a}=\sqrt{\frac{K_{e s}}{J}} \\
& \frac{\omega_{o}(s)}{T_{r}(s)}=\frac{\omega_{a}}{J_{e} s\left(s^{2}+\omega_{n}^{2}\right)}, \omega_{n}=\omega_{a} \sqrt{1+\frac{J}{J_{e}}}
\end{aligned}
$$

The un-damped natural frequencies $\omega_{n}$ and $\omega_{a}$ of the pole and zero pairs in Equation (8) are referred to as the resonant and anti-resonant frequencies [17]. As can be seen, both transfer functions contain an un-damped mode, and hence oscillation may occur if this mode is not adequately damped. With $J_{e} \gg J, \omega_{n}=\omega_{a}$, oscillations occur on the LSR at the resonant frequency $\omega_{n}$ but are filtered by the relatively large equivalent inertia seen by the HSR. With $J_{e} \ll J$, the resonant frequency given by $\omega_{n} \approx \sqrt{K_{e s} / J_{e}}$ is dictated by the equivalent inertia and the equivalent stiffness.

Table 1. PDD parameters.

\begin{tabular}{lc}
\hline$J_{h}\left(\mathrm{kgm}^{2}\right)$ & $3.8 \times 10^{-3}$ \\
$J_{o}\left(\mathrm{kgm}^{2}\right)$ & $2.5 \times 10^{-3}$ \\
$J_{L}\left(\mathrm{kgm}^{2}\right)$ & 0.28 \\
$R_{p}(\Omega)$ & 2 \\
$\varphi_{m}(\mathrm{~Wb})$ & 0.59 \\
$T_{\max }(\mathrm{Nm})$ & 135 \\
$L_{d}(\mathrm{H})$ & $32.6 \times 10^{-3}$ \\
$L_{q}(\mathrm{H})$ & $32.6 \times 10^{-3}$ \\
$B_{h}(\mathrm{Nms} / \mathrm{rad})$ & $1.0 \times 10^{-4}$ \\
$B_{o}(\mathrm{Nms} / \mathrm{rad})$ & $2.0 \times 10^{-4}$ \\
$K_{d}(\mathrm{Nms} / \mathrm{rad})$ & $0.5 \times 10^{-4}$ \\
$\omega_{o_{\max }}(\mathrm{rad} / \mathrm{s})$ & 30 \\
$\omega_{h_{\max }}(\mathrm{rad} / \mathrm{s})$ & 345 \\
$U_{d c}(\mathrm{~V})$ & 435 \\
$i_{q \max }(\mathrm{A})$ & 9 \\
\hline
\end{tabular}

The electrical dynamics of the PDD is similar to that of the conventional surface mounted brushless permanent magnet machine. They are given in term of the $d-q$ axis currents by 


$$
\begin{gathered}
\frac{d i_{d}}{d t}=-\frac{R_{p}}{L_{d}} i_{d}+\frac{\omega_{e} L_{q} i_{q}}{L_{d}}+\frac{v_{d}}{L_{d}} \\
\frac{d i_{q}}{d t}=-\frac{R_{p}}{L_{q}} i_{q}-\frac{\omega_{e} L_{d} i_{d}}{L_{q}}+\frac{v_{q}-K_{e} \omega_{h}}{L_{q}}
\end{gathered}
$$

$L_{d}$ and $L_{q}$ are the d- and q-axis motor inductances, respectively; $R_{p}$ is the motor winding resistance per phase; $K_{t}$ and $K_{e}$ are the motor torque and back-emf constants, respectively. $v_{d}$ and $v_{q}$ are d- and q-axis voltages, respectively, and $\omega_{e}=p \times \omega_{h}$ is the electric angular frequency of the PDD. The parameters of the PDD are given in Table 1.

\section{Complex Frequency Domain Analysis of the Pseudo Direct Drive}

Figure 4 represents the PDD under a generic speed controller. The field oriented control is used to control the currents in the $\mathrm{d}-\mathrm{q}$ axis reference frame. The gains of the PI current controllers for $i_{d}$ and $i_{q}$ are designed for a bandwidth of $400 \mathrm{~Hz}$. The generic speed controller represents one of the following controllers: PI, IP or SFBK controller. A derivation of the system states in closed loop is obtained for each controller structure. The gains of the three speed controllers have been tuned with GA in Simulink to satisfy the ITAE performance index defined in [15] against a complete closed-loop model comprised of the PDD dynamics, a pulse width modulation (PWM) block, the current controllers and the speed controller. The set of gains obtained by GA are used for the frequency domain analysis and time domain simulations as well as for validation of the system in real time implementation.

Figure 4. Schematic of the Pseudo Direct Drive under a speed controller.

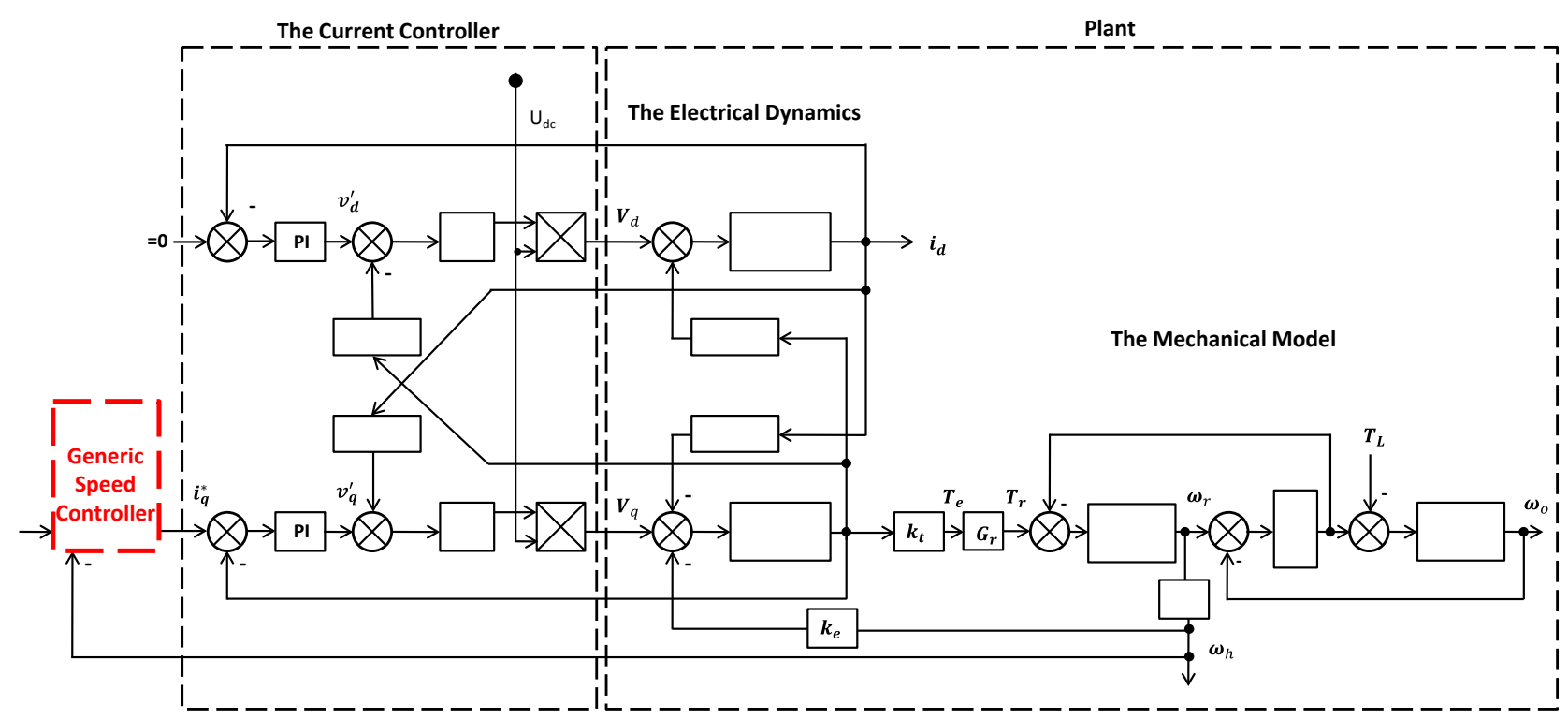

The SFBK structure described in Figure 5 represents one of the generic controllers of the system in Figure 4, where the feedback signal $\omega_{o}$ is taken from the observer. The controller has four proportional gains and one integral gain. Three proportional gains are related to the feedback of the system states, viz., the speed of the HSR, $\omega_{h}$, the speed of the LSR, $\omega_{o}$ and the referred load angle $\theta_{e}$. The fourth gain $K_{s}$ is employed to correct any deviation from synchronization that may occur in the speeds of the 
two rotors. The gains obtained by GA tuning are given in Table 2, full details of GA tuning and implementation of this system is provided in [14].

Table 2. Gains of the implemented controllers.

\begin{tabular}{|c|c|c|}
\hline \multicolumn{3}{|c|}{ Proportional Gain(s) } \\
\hline PI & \multicolumn{2}{|c|}{$K_{p}=0.8386$} \\
\hline IP & \multicolumn{2}{|c|}{$K_{p}=0.3469$} \\
\hline & \multicolumn{2}{|c|}{$K_{\omega h}=1.765$} \\
\hline & \multicolumn{2}{|c|}{$K_{\omega o}=1.699$} \\
\hline SFBK & \multicolumn{2}{|c|}{$K_{\theta_{e}}=9.7856$} \\
\hline & \multicolumn{2}{|c|}{$K_{S}=0.1122$} \\
\hline \multicolumn{2}{|c|}{ Integral Gain } & ITAE \\
\hline PI & $K_{i}=6.863$ & 2.03 \\
\hline IP & $K_{i}=235.01$ & 1.80 \\
\hline SFBK & $K_{i}=5132.8$ & 1.67 \\
\hline \multicolumn{3}{|c|}{ Current Controller Gains } \\
\hline \multicolumn{3}{|c|}{$K_{p q}=K_{p d}=81.93$} \\
\hline \multicolumn{3}{|c|}{$K_{i q}=K_{i d}=5026.5$} \\
\hline
\end{tabular}

Figure 5. State Feedback Controller Structure.

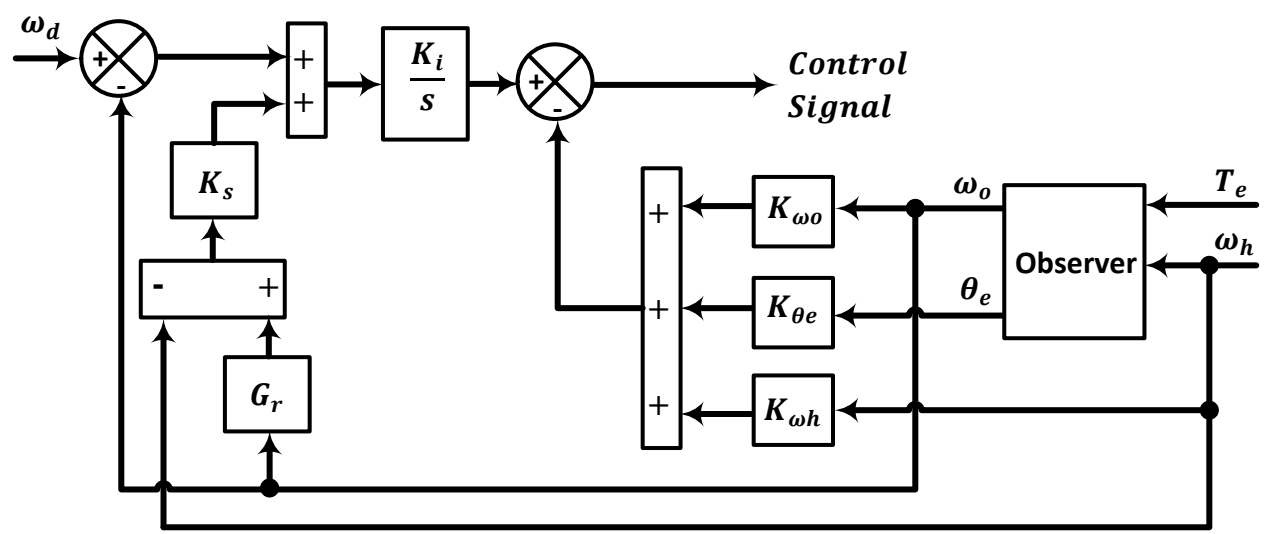

In the frequency domain analysis, the observer dynamics are neglected, as the estimation error of the observer is very small compared to direct measurements. The observer has been linearised around the rated torque of $100 \mathrm{Nm}$ resulting in $\theta_{e} \approx 0.8 \mathrm{rad}$. The design of the observer and sensitivity analysis has been reported in [15].

The closed-loop system may be represented as follows

$$
\dot{\boldsymbol{X}}=f(X, U)
$$

where $\mathbf{X}$ and $\mathbf{U}$ are the vectors of the state variables and inputs, respectively, and $f(\boldsymbol{X}, \boldsymbol{U})$ is the vector of the non-linear functions of $\mathbf{X}$ and $\mathbf{U}$ [18]. They are given by

$$
\boldsymbol{X}=\left[\begin{array}{llllllll}
i_{d} & i_{q} & x_{D} & x_{Q} & x & \omega_{h} & \omega_{o} & \theta_{e}
\end{array}\right]^{T}, U=\omega_{d}
$$


The components of the vector function $f(\boldsymbol{U}, \boldsymbol{X})$ for the SFBK controller are given in Equation (12), where $x_{D}$ and $x_{Q}$ are the internal states of the d- and q-axis current PI controllers, respectively; $x$ is the internal state of the SFBK speed controller. $K_{p d}$ and $K_{p q}$ are proportional gains of the d- and q-axis current controller, respectively; $K_{i d}$ and $K_{i q}$ are the integral gains of the d- and q-axis current controllers, respectively. For a given value of the inputs $\omega_{d}$ it can be shown that in steady state, the operating points of the state variables are obtained as $i_{d 0}=0, x_{D 0}=0, \omega_{h 0}=\omega_{d}, x_{0}=i_{q 0}$, the DC supply voltage, $U_{d c}$, only varies over a small range so the ratio $U_{d c} / U_{d c}^{*} \approx 1$. $i_{d}^{*}$ is set to zero for maximum torque per Ampere operation since flux weakening is not required.

$$
\left.\begin{array}{c}
f_{1}=-\frac{R_{p}}{L_{d}} i_{d}-\frac{K_{p d}}{L_{d}} i_{d}+\frac{1}{L_{d}} x_{D} \\
f_{2}=-\frac{R_{p}}{L_{q}} i_{q}-\frac{K_{p q}}{L_{q}} i_{q}+\frac{1}{L_{q}} x_{Q}+\frac{K_{p q}}{L_{q}} x-\left(\frac{K_{p q} K_{\omega_{h}}}{L_{q}}+\frac{p_{h} \psi_{m}}{L_{q}}\right) \omega_{h}-\frac{K_{\omega_{o}} K_{p q}}{L_{q}} \omega_{o}-\frac{K_{\theta} K_{p q}}{L_{q}} \theta_{e} \\
f_{4}=-K_{i q} i_{q}+x K_{i q}-K_{\omega_{h}} K_{i q} \omega_{h}-K_{\omega_{o}} K_{i q} \omega_{o}-K_{\theta} K_{i q} \theta_{e} \\
f_{5}=K_{i} \frac{\omega_{d}}{G_{r}}-K_{i} K_{s} \omega_{h}+K_{i} K_{s} G_{r} \omega_{o}-K_{i} \omega_{o} \\
f_{6}=\frac{T_{e}}{J_{h}}-\frac{T_{\max }}{J_{h} G_{r}} \sin \left(\theta_{e}\right) \\
f_{7}=\frac{T_{\max }}{J} \sin \left(\theta_{e}\right)-\frac{T_{L}}{J} \\
f_{8}=p_{h} \omega_{h}-n_{s} \omega_{o}
\end{array}\right\}
$$

The state-space equation in Equation (11) can be linearized at the steady-state operating point $\left(\mathbf{X}_{\mathbf{0}}, \mathbf{U}_{\mathbf{0}}\right)$, and the Jacobian matrix of Equation (12) is obtained as follows

$$
\boldsymbol{A}=\left.\frac{\partial f(X, U)}{\partial X}\right|_{\substack{X=X_{0} \\ U=U_{0}}} \quad \boldsymbol{B}=\left.\frac{\partial f(X, U)}{\partial U}\right|_{\substack{X=X_{0} \\ U=U_{0}}}
$$

The matrices $\mathbf{A}, \mathbf{B}, \mathbf{C}$ and $\mathbf{D}$ are given by

$$
\boldsymbol{A}=\left[\begin{array}{cccccccc}
-\frac{R+K_{p d}}{L_{d}} & 0 & \frac{1}{L_{d}} & 0 & 0 & 0 & 0 & 0 \\
0 & -\frac{R+K_{p q}}{L_{q}} & 0 & \frac{1}{L_{q}} & \frac{K_{p q}}{L_{q}} & -\frac{K_{p q} K_{\omega_{h}}+p_{h} \psi_{m}}{L_{q}} & -\frac{K_{p q} K_{\omega_{o}}}{L_{q}} & -\frac{K_{p q} K_{\theta_{e}}}{L_{q}} \\
-K_{i d} & 0 & 0 & 0 & 0 & 0 & 0 & 0 \\
0 & -K_{i q} & 0 & 0 & K_{i q} & -K_{i q} K_{\omega_{h}} & -K_{i q} K_{\omega_{o}} & -K_{i q} K_{\theta_{e}} \\
0 & 0 & 0 & 0 & 0 & -K_{i} K_{s} & -K_{i}+K_{i} K_{s} G_{r} & 0 \\
0 & \frac{K_{t}}{J_{h}} & 0 & 0 & 0 & 0 & 0 & -\frac{T_{\max }}{J_{h} G_{r}} \cos \left(\theta_{e}\right) \\
0 & 0 & 0 & 0 & 0 & 0 & 0 & \frac{T_{\max }}{J} \cos \left(\theta_{e}\right) \\
0 & 0 & 0 & 0 & 0 & p_{h} & -n_{s} & 0
\end{array}\right]
$$




$$
\boldsymbol{B}=\left[\begin{array}{c}
0 \\
0 \\
0 \\
0 \\
K_{i} \\
0 \\
0 \\
0
\end{array}\right] \quad \boldsymbol{C}=\left[\begin{array}{l}
0 \\
0 \\
0 \\
0 \\
0 \\
1 \\
0 \\
0
\end{array}\right]^{T} \quad \boldsymbol{D}=[0]
$$

The state-space equations of the drive system with the IP and PI structures shown in Figures 6 and 7 are obtained by replacing the generic controller of the system in Figure 4. The speed and position feedback signals are directly measured from a resolver mounted on the HSR.

Figure 6. Integral and proportional controller structure.

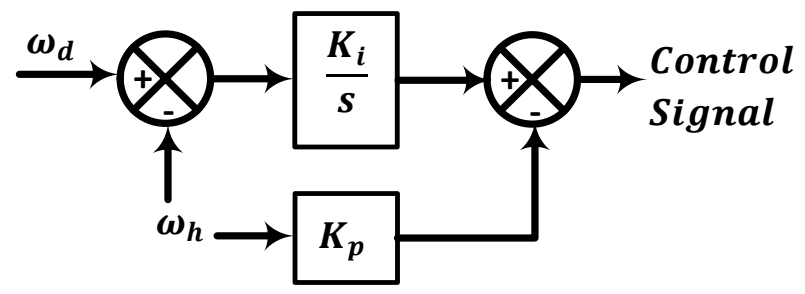

Figure 7. Proportional and integral controller structure.

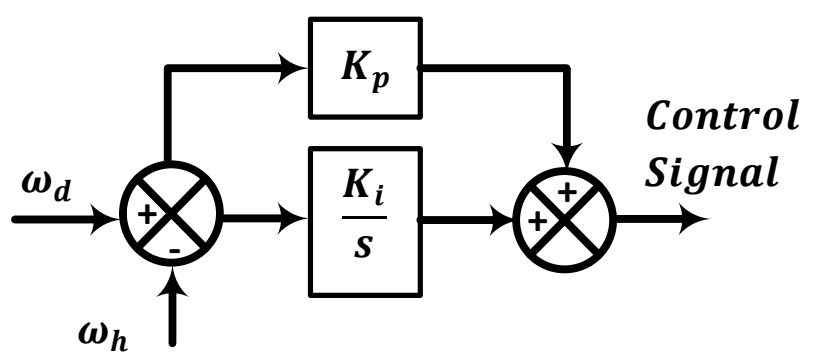

A detailed derivation and the resulting equations of the PI and IP controllers are given in [19].

From the linearized state-space equations, closed-loop transfer functions between the speed demand and speed output can be obtained and their poles and zeros are explicitly plotted in Figure 8, where the poles are marked in " $x$ ” and zeros in "o". The overlapping poles on the negative real axis close to the origin are related to the electric time constant of the d- and q- axis currents. Their effects are cancelled by two zeros through the pole/zero cancellation in the current controller design. The complex conjugate poles-pair with great negative real is associated with the interaction of the SFBK control with the mechanical dynamics of the PDD. However, the dominant pole-pair of the closed loop system is the complex conjugate pole-pair with far less negative real and the imaginary close to the torsional resonant frequency given in Equation (8). The complex pair of zeros, representing the anti-resonant frequency, is very close to the dominant poles. The load torque, motor/load inertia, gear ratio, magnetic damping and the stiffness of the magnetic gear influence the location of the dominant pole-pair and hence the dynamic behaviour of the closed loop drive system. 
Figure 8. Poles and zeros distribution for $G_{1}(s)=\frac{\omega_{h}}{\omega_{d}}$.

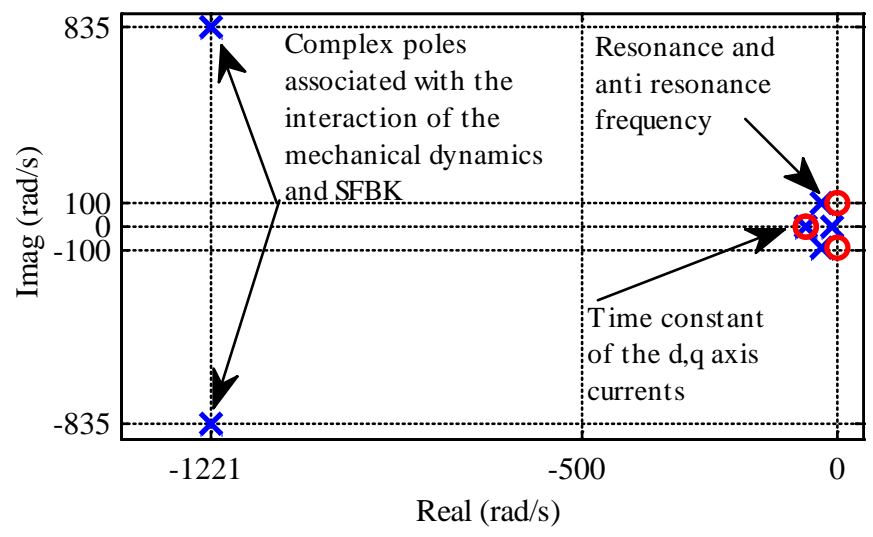

Figure 9 shows the poles/zeros of the transfer function $G_{2}(s)$, where the distribution is similar to $G_{1}(s)$ in Figure 8 except the zeros at the anti-resonant frequency is not present in $G_{2}(s)$ in accordance with Equation (8). Pole-zero distributions associated with IP and PI controllers have a similar pattern and therefore not plotted. Their dominant poles are also associated with the torsional resonance. In order to compare the performance of three optimally designed controllers for suppression of the torsional oscillation, the dominant poles which result from the three controllers are plotted together in the same s-plane.

Figure 9. Poles and zeros distribution for $G_{2}(s)=\frac{\omega_{0}}{\omega_{d}}$.

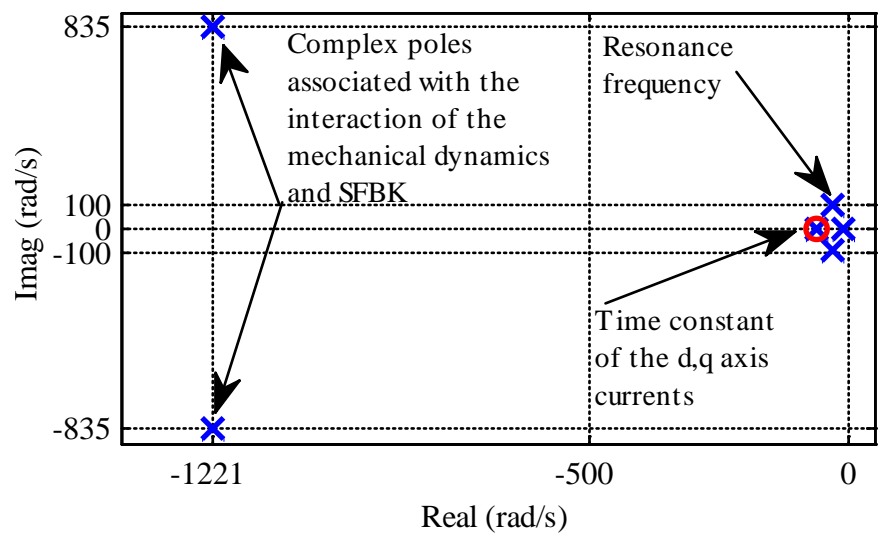

Figure 10 shows the dominant poles of the three controllers in closed loop. It is evident that much more damping has been achieved with the SFBK controller. By using a well-tuned controller the poles are placed such that the ITAE is minimised, hence optimum damping and bandwidth is achieved. This also confirms the time domain simulation results that show the system having achieved more damping by reducing the oscillations in the output.

In contrast, the PI and IP control structure results in the dominant poles being close to the imaginary axis and hence much lower damping. The IP can only achieve a maximum damping of $\zeta=0.13$ and the PI exhibit very poor damping of $\zeta=0.023$, being an order of magnitude lower in comparison with the damping obtained by the SFBK controller. Hence both the PI and IP structures are not capable of improving the damping of the dominant pole-pair associated with torsional resonance in the PDD dynamics as compared to the SFBK. 
Figure 10. The dominant poles for all controllers.

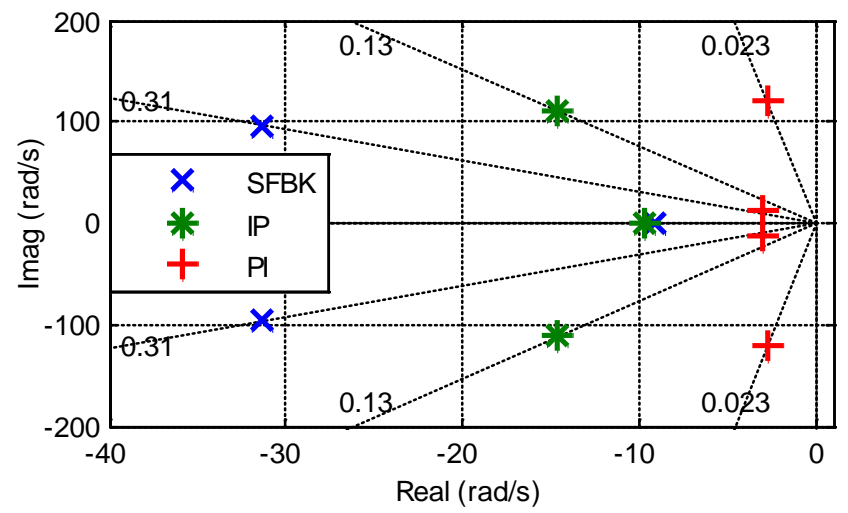

Figure 11 shows the loci of the dominant pole-pair with increase in the load torque $T_{L}$ from 0 to the maximum load in 3 steps ( $0 \mathrm{Nm}, 50 \mathrm{Nm}, 100 \mathrm{Nm}$ ). As will be seen, the SFBK control exhibits robust performance against the load torque variations as the system damping increases and bandwidth is nearly constant with increase in load torque. An increase in the load torque seems to slightly increase the system damping under the PI albeit its damping is still significantly low. The load torque increase did not affect the system damping under IP controller. However, the bandwidth has been reduced by more than $15 \%$ with the load torque increase.

Figure 11. Loci of dominant poles with load torque variation for PI, IP and SFBK controllers.

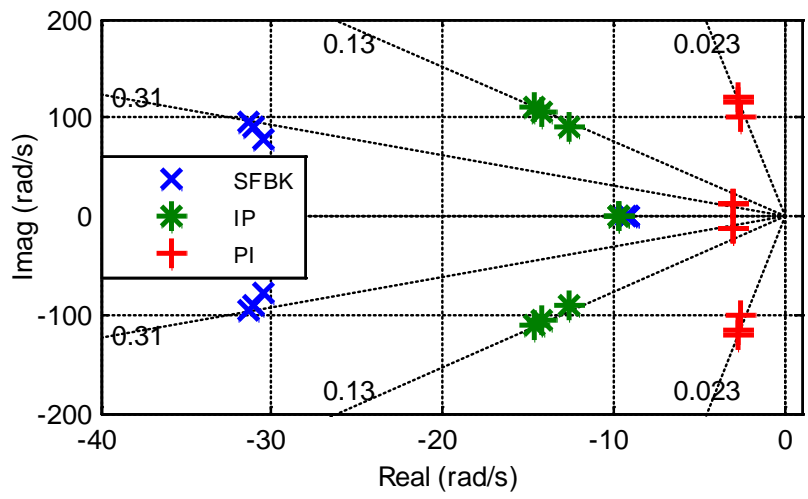

Figure 12 shows the dominant poles of the three controllers when subjected to a load inertia variation, where the load to motor inertia ratio $R$ is given by

$$
R=\frac{J}{J_{e}}
$$

By changing the load inertia in four steps, $R$ varies from $0.25,0.56,1$ to 2 . It can be seen that with the PI control, both the system damping and control bandwidth decrease with decrease in the inertia ratio. The damping and bandwidth are still very poor even when the ratio is increased to $R=2$.

The dominant poles of the closed loop system under the IP control show a high level of sensitivity to the inertia variation, and any decrease in $R$ results in significant reduction in the damping. More specifically, the figure shows that the system damping can be reduced by an order of magnitude and the bandwidth by more than half when $R$ is varied from 0.56 to 0.125 . Also the bandwidth may be reduced significantly when $R$ is increased to $R=2$ as the two real poles representing the d-q axis 
currents are pushed further towards the origin reducing the system bandwidth as shown with a green arrow.

Figure 12. Loci of dominant poles with load inertia variation for PI, IP and SFBK controllers.

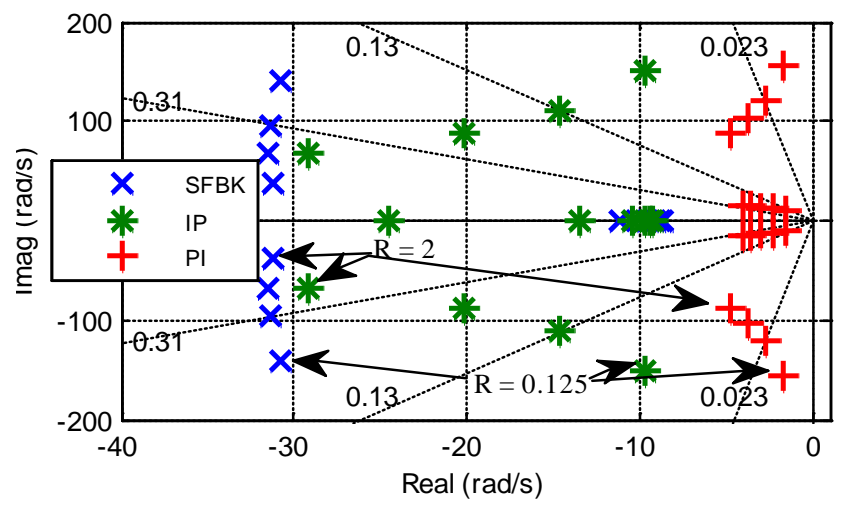

The SFBK controller is much robust than the other two controllers. It has been seen that the lowest damping which results when $R=0.125$ is 0.14 , is still more than an order of magnitude greater than the damping achieved by the PI control, and much greater than that of the IP control. Moreover, the SFBK control bandwidth is insensitive to the inertia ratio variations. Therefore, the system with the SFBK control exhibits more robustness towards parameter variations.

\section{Experimental Results and Discussion}

The findings of the above analysis have been validated with a specially designed and built test rig as shown in Figure 13. The PDD control, including the PI, IP and, the SFBK and observer, is implemented in a dSPACE real-time hardware platform at sampling frequency of $10 \mathrm{kHz}$ with PWM of the drive machine set at $8 \mathrm{kHz}$. The resulting torque command is fed to a commercial drive in torque control mode. The PDD operates in speed control mode and is loaded by the PM machine in torque control mode. The PM load machine is coupled to the PDD via a 10:1 inline gear box in order to provide sufficient load torque for the PDD operation. The speed/position of the HSR is measured with a resolver, and an encoder is placed on the LSR for the purpose of monitoring its speed/position only. The load torque is inferred from the current in the load machine and the relationship between the current and the torque has been determined using torque transducer.

Figure 13. The Pseudo Direct Drive test Rig.

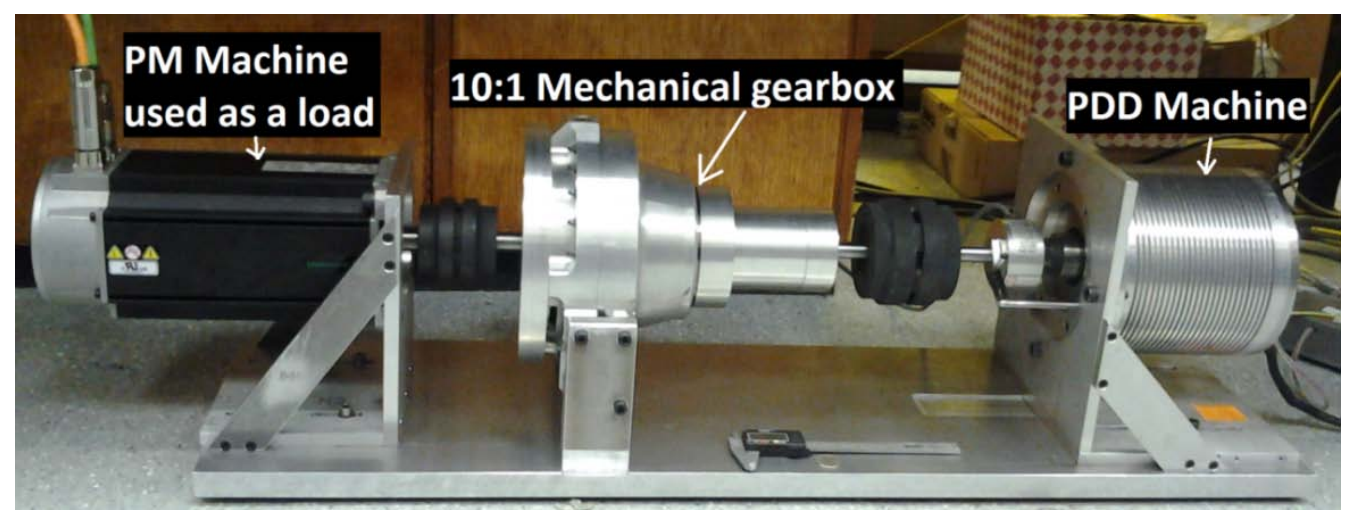


To test the speed response with one of the controllers PI, IP, and SFBK, the LSR of the PDD is accelerated from standstill to $100 \mathrm{rpm}$, and at $2 \mathrm{~s}$ a load torque of $100 \mathrm{Nm}$ is applied by the load machine for duration of $3 \mathrm{~s}$. Simulated and experimental results are shown in Figures 14-16 for the three controllers together with the load torque waveform.

Figure 14 shows the simulation and measured PDD responses under the PI control. It can be seen that whilst the simulated and measured speed responses agree very well, undesirable oscillations which result from the poor damping in both rotor speeds are very significant.

Figure 14. PDD speed responses under PI. (a) Simulated; (b) Measured.

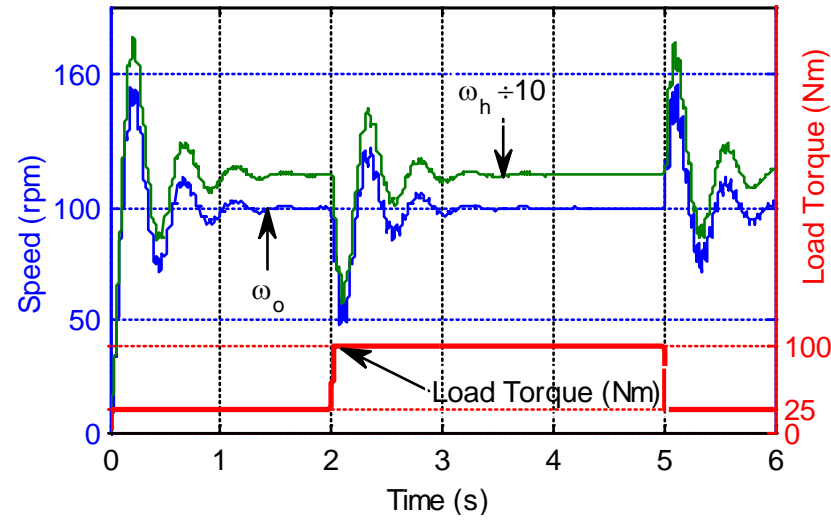

(a)

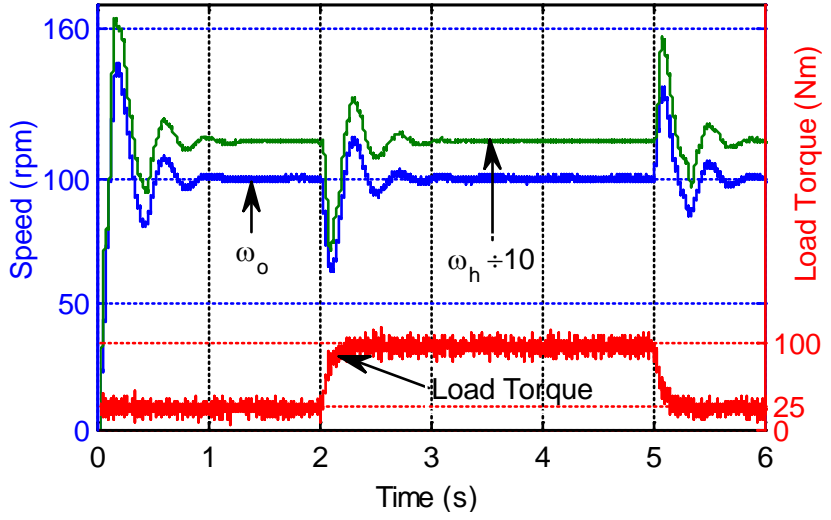

(b)

Figure 15 shows the PDD speed responses under the IP controller, where an improved speed tracking and disturbance rejection is achieved in comparison with the PI control. However, oscillations still appear when the load is applied or removed.

Figure 15. PDD responses under IP. (a) Simulated; (b) Measured.

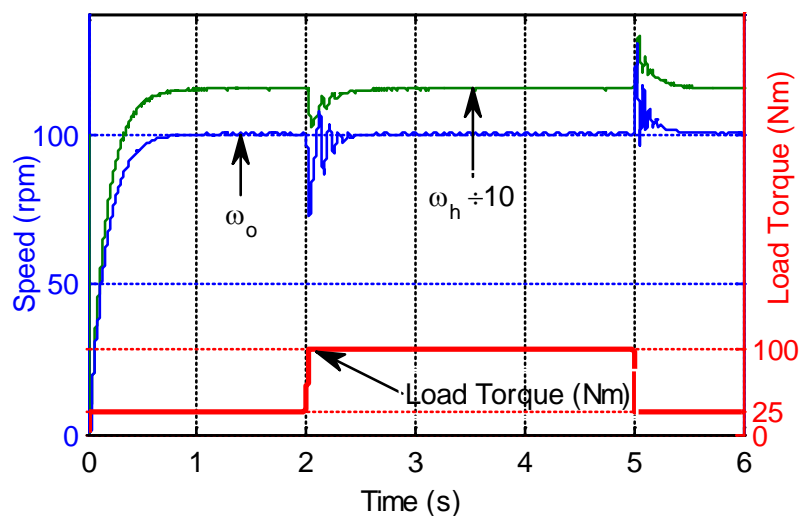

(a)

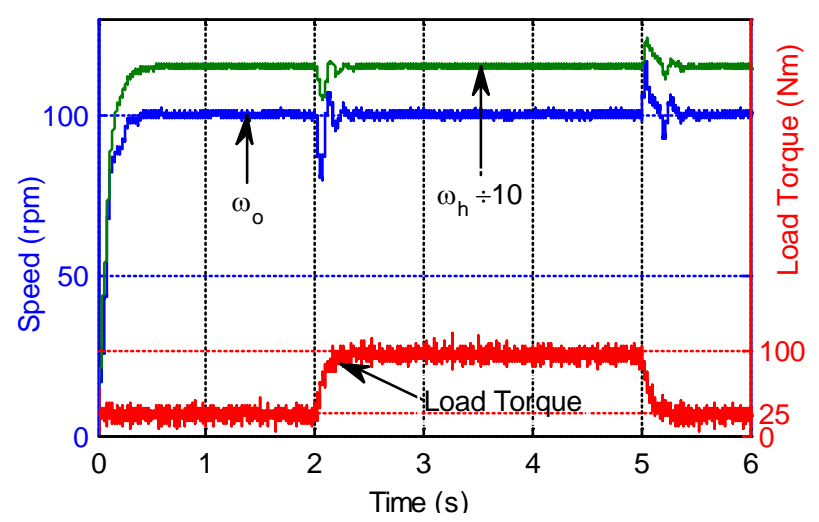

(b)

Figure 16 shows the response of the PDD under SFBK control, where the controller exhibits good disturbance rejection and speed tracking, with no visible oscillations in the output.

The SFBK is realized using a reduced order observer. Figure 17 shows the measured speed $\omega_{o}$ against the estimated speed $\widehat{\omega}_{o}$ from the reduced order observer in real time. 
Figure 16. PDD responses under SFBK. (a) Simulated; (b) Measured.

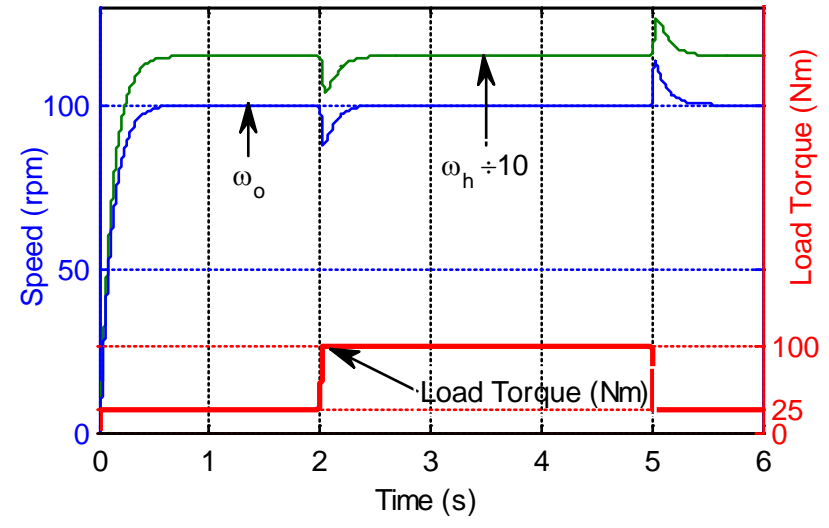

(a)

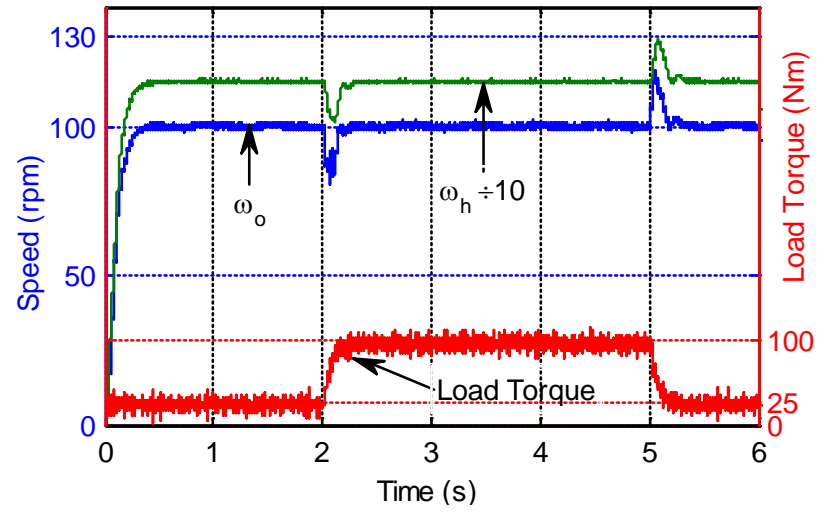

(b)

Figure 17. Measured and estimated LSR and HSR speeds.

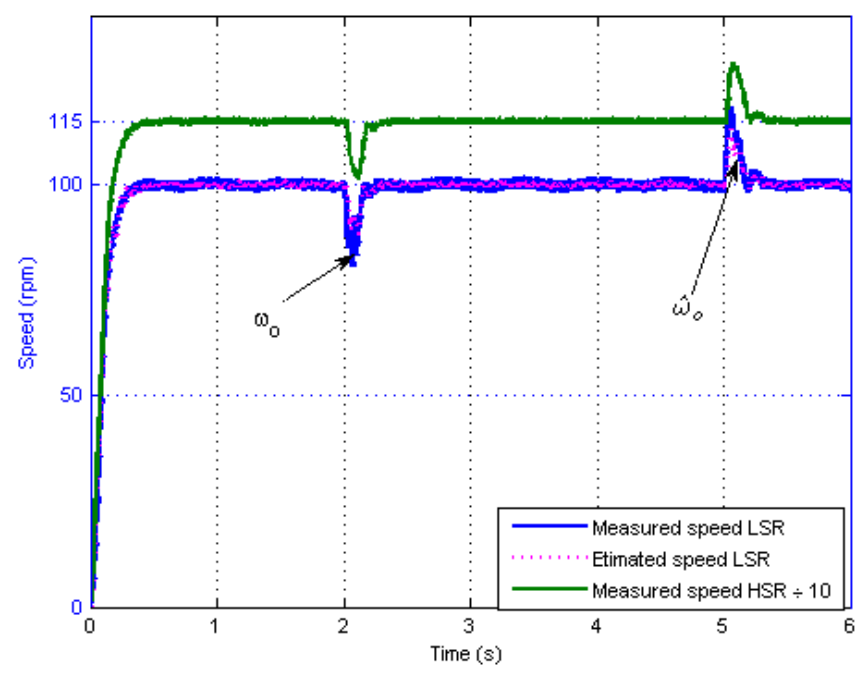

Figures 18-20 show the measured d-q axis currents of the three controllers PI, IP and SFBK.

Figure 18. Measured currents $\mathrm{i}_{\mathrm{q}}$ and $\mathrm{i}_{\mathrm{d}}$ under PI control.

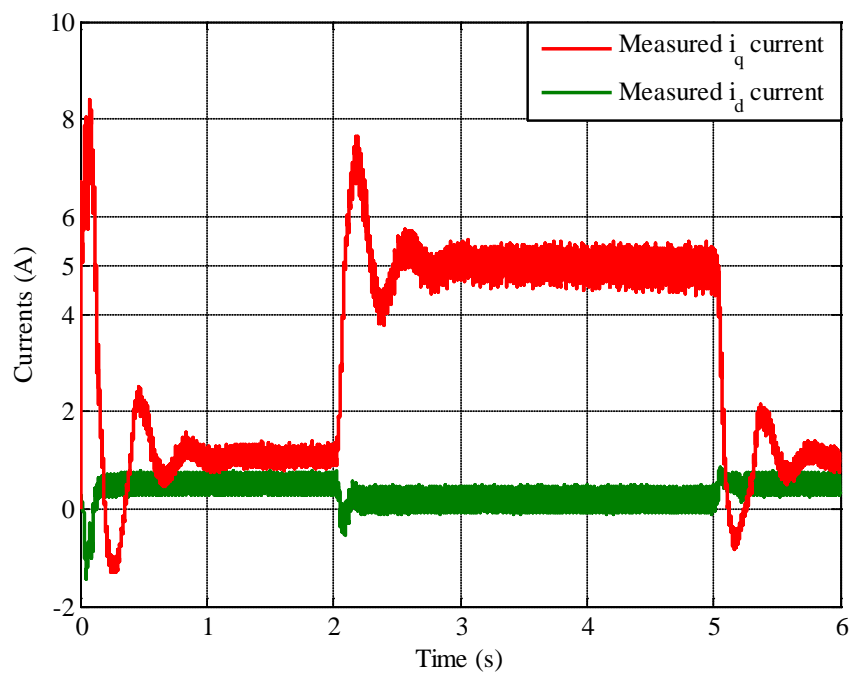


Figure 19. Measured currents $i_{q}$ and $i_{d}$ under IP control.

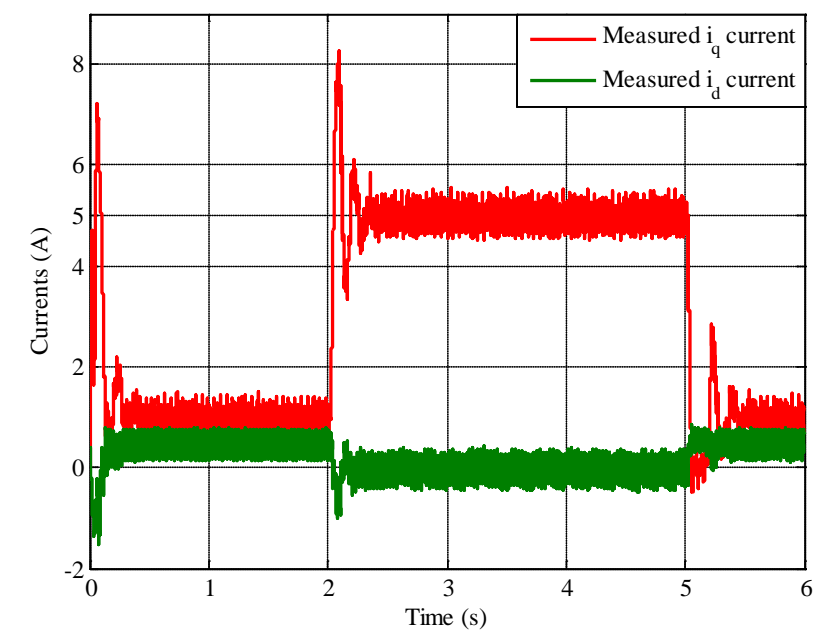

Figure 20. Measured currents $i_{q}$ and $i_{d}$ under SFBK control.

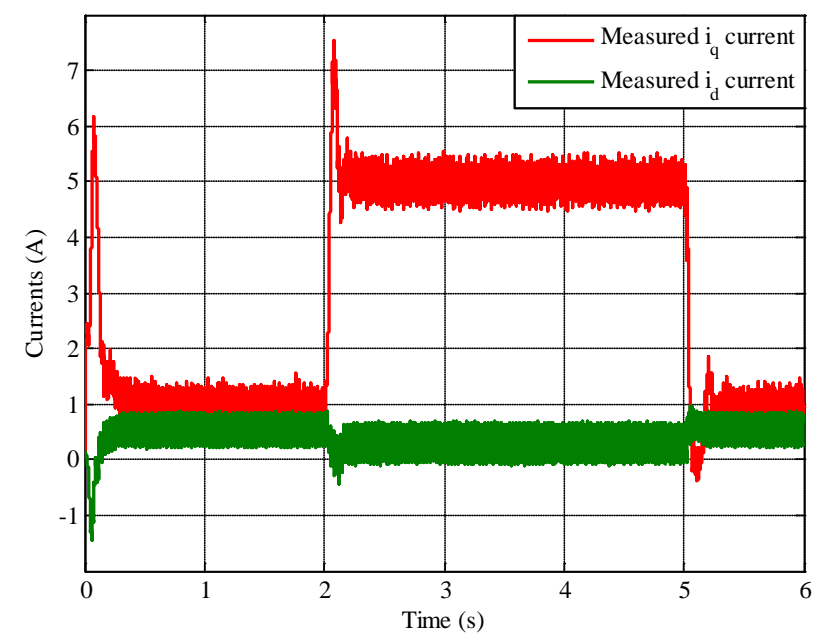

Figures 21 and 22 show the PDD operated at \pm 50 and \pm 150 rpm with load torque of 100 and $50 \mathrm{Nm}$ applied to the LSR from time 2 to $5 \mathrm{~s}$ and 10 to $13 \mathrm{~s}$ respectively.

Figure 21. LSR under SFBK control and $100 \mathrm{Nm}$ load torque.

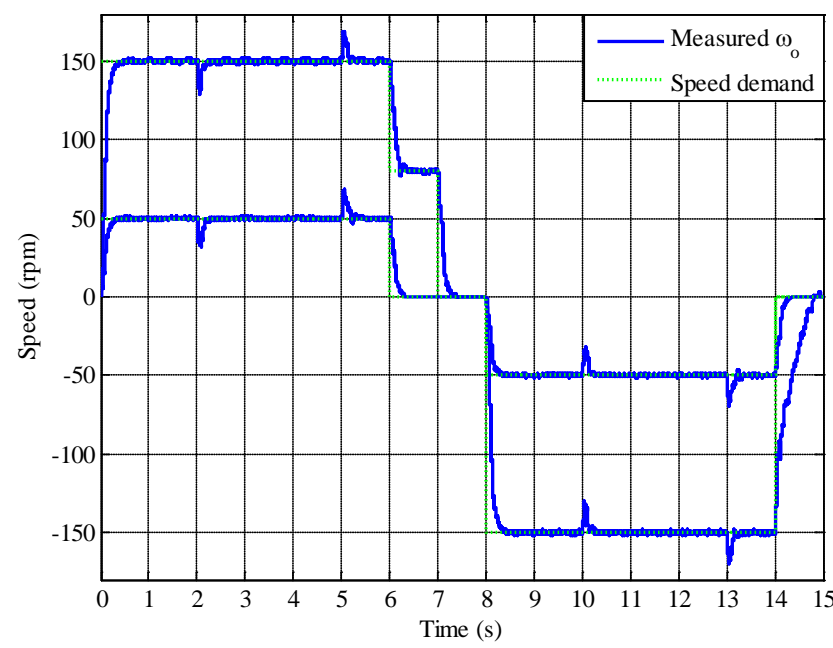


Figure 22. LSR under SFBK control and $50 \mathrm{Nm}$ load torque.

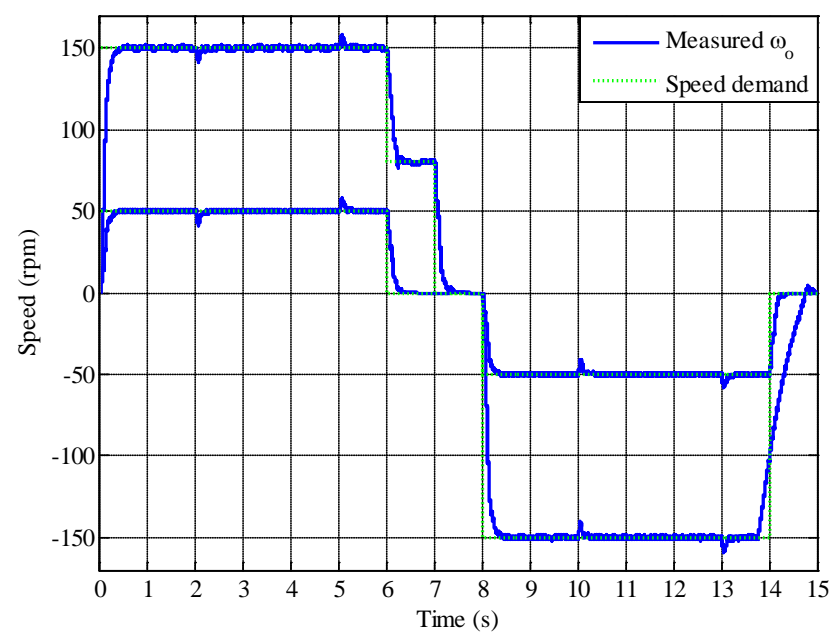

\section{Conclusions}

The complex frequency domain analysis has provided an in-depth understanding of the behaviour of the Pseudo Direct Drive with three candidate controllers. A closed loop model of the PDD under three controller structures PI, IP, SFBK has been established and the influence of the control structures and parameters, such as the load torque, load inertia on the system damping and the controller bandwidth have been investigated. It has been shown that the state feedback controller tuned with a genetic algorithm provides the best damping and robustness to parameter changes and uncertainties compared to the PI and IP. Experimental results have validated the findings of the theoretical analysis.

\section{Acknowledgments}

The work was carried out with the support of the UK EPSRC, and Magnomatics Ltd through the provision of a $\mathrm{PhD}$ studentship.

\section{Author Contributions}

The authors have compared the performance of three candidate speed controllers for a pseudo direct drive. All the controllers have been tuned using genetic algorithm to guarantee optimum performance and the cause of oscillation in the speed output with PI and IP controllers has been identified. The measure to dampen the oscillation has been proposed and experimentally demonstrated.

The authors have also presented a systematic way of studying complex systems in closed loop control in frequency domain, where the performance of various controllers could be quickly analyzed using the derived matrices and the drive system parameters.

\section{Conflicts of Interest}

The authors declare no conflict of interest. 


\section{References}

1. Powell, D.J.; Calverley, S.D.; de Wildt, F.; Daffey, K. Design and analysis of a Pseudo Direct-Drive propulsion motor. In Proceedings of the 5th IET International Conference on Power Electronics, Machines and Drives (PEMD 2010), Brighton, UK, 19-21 April 2010; pp. 1-2.

2. Atallah, K.; Rens, J.; Mezani, S.; Howe, D. A Novel 'Pseudo' Direct-Drive Brushless Permanent Magnet Machine. IEEE Trans. Magn. 2008, 44, 4349-4352.

3. Shuangxia, N.; Ho, S.L.; Fu, W.N.; Wang, L.L. Quantitative Comparison of Novel Vernier Permanent Magnet Machines. IEEE Trans. Magn. 2010, 46, 2032-2035.

4. Li, J.; Wang, J.; Zhigang, Z.; Yan, W. Analytical analysis and implementation of a low-speed high-torque permanent magnet vernier in-wheel motor for electric vehicle. J. Appl. Phys. 2012, 111, $07 \mathrm{E} 727$.

5. Harris, M.R.; Pajooman, G.H.; Sharkh, S.M.A. The problem of power factor in VRPM (transverse-flux) machines. In Proceedings of the 1997 Eighth International Conference on Electrical Machines and Drives, Cambridge, UK, 1-3 September 1997; pp. 386-390.

6. Bouheraoua, M.; Wang, J.; Atallah, K. Slip recovery and prevention in Pseudo Direct Drive permanent magnet machines. In Proceedings of the 2013 IEEE Energy Conversion Congress and Exposition (ECCE), Denver, CO, USA, 15-19 September 2013; pp. 2162-2169.

7. O’Sullivan, T.M.; Bingham, C.M.; Schofield, N. High-Performance Control of Dual-Inertia Servo-Drive Systems Using Low-Cost Integrated SAW Torque Transducers. IEEE Trans. Ind. Electron. 2006, 53, 1226-1237.

8. Zhang, G. Speed control of two-inertia system by PI/PID control. IEEE Trans. Ind. Electron. 2000, 47, 603-609.

9. Hori, Y.; Sawada, H.; Yeonghan, C. Slow resonance ratio control for vibration suppression and disturbance rejection in torsional system. IEEE Trans. Ind. Electron. 1999, 46, 162-168.

10. Montague, R.G.; Bingham, C.M.; Atallah, K. Characterisation and modelling of magnetic couplings and gears for servo control systems. In Proceedings of the 5th IET International Conference on Power Electronics, Machines and Drives (PEMD 2010), Brighton, UK, 19-21 April 2010; pp. 1-6.

11. Montague, R.; Bingham, C.; Atallah, K. Servo Control of Magnetic Gears. IEEE/ASME Trans. Mechatron. 2012, 17, 269-278.

12. Wang, J.; Atallah, K. Modeling and control of 'pseudo' direct-drive brushless permanent magnet machines. In Proceedings of the 2009 IEEE International Electric Machines and Drives Conference, (IEMDC '09), Miami, FL, USA, 3-6 May 2009; pp. 870-875.

13. Jun-Keun, J.; Seung-Ki, S. Kalman filter and LQ based speed controller for torsional vibration suppression in a 2-mass motor drive system. IEEE Trans. Ind. Electron. 1995, 42, 564-571.

14. Bouheraoua, M.; Wang, J.; Atallah, K. Observer based state feedback controller design for Pseudo Direct Drive using genetic algorithm. In Proceedings of the 6th IET International Conference on Power Electronics, Machines and Drives (PEMD 2012), Bristol, UK, 27-29 March 2012; pp. 1-6.

15. Bouheraoua, M.; Wang, J.; Atallah, K. Design and implementation of an observer-based state feedback controller for a pseudo direct drive. IET Electr. Power Appl. 2013, 7, 643-653. 
16. Chipperfield, A.J.; Fleming, P.J. The MATLAB genetic algorithm toolbox. In Proceedings of the IEE Colloquium on Applied Control Techniques Using MATLAB, London, UK, 26 January 1995; pp. 10/1-10/4.

17. Vukosavic, S.N.; Stojic, M.R. Suppression of torsional oscillations in a high-performance speed servo drive. IEEE Trans. Ind. Electron. 1998, 45, 108-117.

18. Wang, J.; Griffo, A.; Han, L.; Howe, D. Input Admittance Characteristics of Permanent Magnet Brushless AC Motor Drive Systems. In Proceedings of the 2007 IEEE Vehicle Power and Propulsion Conference (VPPC 2007), Arlington, TX, USA, 9-12 September 2007; pp. 191-196.

19. Bouheraoua, M.; Wang, J.; Atallah, K. A complex frequency domain analysis of a closed loop controlled pseudo direct drive. In Proceedings of the 2012 XXth International Conference on Electrical Machines (ICEM), Marseille, France, 2-5 September 2012; pp. 2428-2434.

(C) 2014 by the authors; licensee MDPI, Basel, Switzerland. This article is an open access article distributed under the terms and conditions of the Creative Commons Attribution license (http://creativecommons.org/licenses/by/3.0/). 